9th A. Friedmann International Seminar and

3rd Casimir Symposium 2015

International Journal of Modern Physics: Conference Series

Vol. 41 (2016) 1660120 (11 pages)

(C) The Author(s)

DOI: $10.1142 /$ S2010194516601204

\title{
Regular rotating electrically charged structures in nonlinear electrodynamics minimally coupled to gravity
}

\author{
Irina Dymnikova \\ A. F. Ioffe Physico-Technical Institute, Politekhnicheskaja 26, 194021 St.Petersburg, Russia \\ University of Warmia and Mazury, Stoneczna 54, 10-710 Olsztyn, Poland \\ irina@uwm.edu.pl \\ Evgeny Galaktionov \\ A. F. Ioffe Physico-Technical Institute, Politekhnicheskaja 26, 194021 St.Petersburg, Russia \\ evgalakt@mail.ru
}

Received 22 September 2015

Published 18 March 2016

\begin{abstract}
In nonlinear electrodynamics minimally coupled to gravity, regular spherically symmetric electrically charged solutions satisfy the weak energy condition and have obligatory de Sitter center. By the Gürses-Gürsey algorithm they are transformed to regular axially symmetric solutions asymptotically Kerr-Newman for a distant observer. Rotation transforms de Sitter center into de Sitter equatorial disk embedded as a bridge into a de Sitter vacuum surface. The de Sitter surfaces satisfy $p=-\rho$ and have properties of a perfect conductor and ideal diamagnetic. The Kerr ring singularity is replaced with the superconducting current which serves as a non-dissipative electromagnetic source of the asymptotically Kerr-Newman geometry. Violation of the weak energy condition is prevented by the basic requirement of electrodynamics of continued media.
\end{abstract}

Keywords: Regular black hole; electromagnetic spinning soliton; nonlinear electrodynamics coupled to gravity.

PACS numbers: 04.70.Bw; 04.70.-s; 04.20.Dw; 04.20.Jb

\section{Introduction}

The Kerr-Newman solution to the source-free Maxwell-Einstein equations reads ${ }^{1}$

$$
\begin{aligned}
d s^{2}= & \frac{\left(2 m r-e^{2}\right)-\Sigma}{\Sigma} d t^{2}+\frac{\Sigma}{\Delta} d r^{2}+\Sigma d \theta^{2}-\frac{2 a\left(2 m r-e^{2}\right) \sin ^{2} \theta}{\Sigma} d t d \phi \\
& +\left(r^{2}+a^{2}+\frac{\left(2 m r-e^{2}\right) a^{2} \sin ^{2} \theta}{\Sigma}\right) \sin ^{2} \theta d \phi^{2} ; \quad \Delta=r^{2}-2 m r+a^{2}+e^{2},
\end{aligned}
$$

This is an Open Access article published by World Scientific Publishing Company. It is distributed under the terms of the Creative Commons Attribution 4.0 (CC-BY) License. Further distribution of this work is permitted, provided the original work is properly cited. 
where $m, e$ and $a$ are the mass, electrical charge and angular momentum, respectively. The function $\Sigma$ and the associated electromagnetic potential read

$$
\Sigma=r^{2}+a^{2} \cos ^{2} \theta ; \quad A_{i}=-\frac{e r}{\Sigma}\left[1 ; 0,0,-a \sin ^{2} \theta\right]
$$

This solution was obtained from the Reissner-Nordström electrovacuum solution with using the complex coordinate transformation found by Newman and Janis. ${ }^{2}$

The Kerr-Newman geometry implies the value of the gyromagnetic ratio $g=2$, predicted for a spinning particle by the Dirac equation. ${ }^{3}$ In the case corresponding to a particle, $a^{2}+e^{2}>m^{2}$, there are no Killing horizons, the manifold is geodesically complete (except for geodesics which reach the singularity) and any point can be connected to any other point by both a future and a past directed time-like curve. Closed time-like curves originate in the region where $g_{\phi \phi}<0$, can extend over the whole manifold and cannot be removed by taking a covering space. ${ }^{3}$

The source models for the Kerr-Newman exterior involving a screening or covering of causally dangerous region, are typically constructed by matching a hypothetical interior material source to the Kerr-Newman solution (for a recent review see Refs. 4 and 5). The problem of matching does not have a unique solution, since one is free to choose arbitrarily the boundary between the exterior and the interior. ${ }^{6}$

On the other hand, basic properties of regular rotating charged objects can be found by analysis of dynamical equations given by nonlinear electrodynamics coupled to gravity (NED-GR). NED theories appear as low-energy effective limits in certain models of string/M-theories. ${ }^{7-9}$ Source-free NED-GR equations admit regular causally safe axially symmetric asymptotically Kerr-Newman solutions, ${ }^{10}$ which describe regular rotating charged black holes and electromagnetic spinning solitons.

The key point is that for any gauge-invariant Lagrangian $\mathcal{L}(F)$, stress-energy tensor of electromagnetic field in the spherically symmetric case has the algebraic structure such that

$$
T_{t}^{t}=T_{r}^{r} \quad\left(p_{r}=-\rho\right) .
$$

Regular spherically symmetric solutions to the Einstein equations with a source term specified by (3) satisfying the weak energy condition (non-negativity of density as measured by any local observe), have obligatory de Sitter center with $p=-\rho .^{11-14}$ Regular spherical NED-GR solutions always satisfy the weak energy condition. ${ }^{5}$ They belong to the Kerr-Schild class ${ }^{15-17}$ and can be transformed by the GürsesGürsey algorithm ${ }^{18}$ into regular axially symmetric solutions, asymptotically KerrNewman for a distant observer. ${ }^{10,19}$ Rotation transforms the de Sitter center into de Sitter vacuum disk which has properties of a perfect conductor and ideal diamagnetic and displays superconducting behavior within a single spinning object. ${ }^{10,20}$ The mass $m$ of an object is generically related to de Sitter vacuum and breaking of space-time symmetry from the de Sitter group in the origin. ${ }^{13}$

In this talk we outline the basic generic features of regular rotating electrically charged objects in nonlinear electrodynamics minimally coupled to gravity. 


\section{Basic Equations}

Nonlinear electrodynamics minimally coupled to gravity is described by the action

$$
S=\frac{1}{16 \pi G} \int d^{4} x \sqrt{-g}[R-\mathcal{L}(F)] ; \quad F=F_{\mu \nu} F^{\mu \nu},
$$

where $R$ is the scalar curvature, and $F_{\mu \nu}=\partial_{\mu} A_{\nu}-\partial_{\nu} A_{\mu}$ is the electromagnetic field. The gauge-invariant electromagnetic Lagrangian $\mathcal{L}(F)$ is an arbitrary function of $F$ which should have the Maxwell limit, $\mathcal{L} \rightarrow F, \mathcal{L}_{F} \rightarrow 1$ in the weak field regime.

Variation with respect to $A^{\mu}$ and $g_{\mu \nu}$ yields the dynamic field equations

$$
\begin{gathered}
\nabla_{\mu}\left(\mathcal{L}_{F} F^{\mu \nu}\right)=0 ; \\
\nabla_{\mu}{ }^{*} F^{\mu \nu}=0 ; \quad{ }^{\star} F^{\mu \nu}=\frac{1}{2} \eta^{\mu \nu \alpha \beta} F_{\alpha \beta} ; \quad \eta^{0123}=-\frac{1}{\sqrt{-g}},
\end{gathered}
$$

where Greek indices run from 0 to 3 and $\mathcal{L}_{F}=d \mathcal{L} / d F$, and the Einstein equations $G_{\mu \nu}=-\kappa T_{\mu \nu}$ with the stress-energy tensor

$$
\kappa T_{\nu}^{\mu}=-2 \mathcal{L}_{F} F_{\nu \alpha} F^{\mu \alpha}+\frac{1}{2} \delta_{\nu}^{\mu} \mathcal{L} ; \quad \kappa=8 \pi G
$$

NED-GR equations do not admit regular spherically symmetric solutions with the Maxwell center, ${ }^{21}$ but they admit regular solutions with the de Sitter center in which field tension goes to zero, while the energy density of the electromagnetic vacuum $T_{t}^{t}$ achieves its maximal finite value which represents the de Sitter cutoff on the self-energy density. ${ }^{22}$ The question of correct description of NED-GR regular electrically charged structures by the Lagrange dynamics is considered in Ref. 23. Regular spherical solutions satisfying (3) are described by the metric

$$
d s^{2}=g(r) d t^{2}-\frac{d r^{2}}{g(r)}-r^{2} d \Omega^{2} ; g(r)=1-\frac{2 \mathcal{M}(r)}{r} ; \mathcal{M}(r)=4 \pi \int_{0}^{r} \tilde{\rho}(x) x^{2} d x,
$$

with the electromagnetic density $\tilde{\rho}(r)=T_{t}^{t}(r)$ from $(7)$. This metric has the de Sitter asymptotic as $r \rightarrow 0$ and the Reissner-Nordström asymptotic as $r \rightarrow \infty$. $^{22}$

Spherically symmetric solutions of the Einstein equations specified by (3) belong to the Kerr-Schild class. ${ }^{16,17}$ By the Gürses-Gürsey algorithm they can be transformed into axially symmetric regular solutions describing rotating objects. ${ }^{18}$

In the Boyer-Lindquist coordinates the Gürses-Gürsey metric reads

$$
d s^{2}=\frac{2 f-\Sigma}{\Sigma} d t^{2}+\frac{\Sigma}{\Delta} d r^{2}+\Sigma d \theta^{2}-\frac{4 a f \sin ^{2} \theta}{\Sigma} d t d \phi+\left(r^{2}+a^{2}+\frac{2 f a^{2} \sin ^{2} \theta}{\Sigma}\right) \sin ^{2} \theta d \phi^{2},
$$

where the Lorentz signature is $[-+++]$, and

$$
\Delta(r)=r^{2}+a^{2}-2 f(r) ; \quad \Sigma=r^{2}+a^{2} \cos ^{2} \theta .
$$

A function $f(r)=r \mathcal{M}(r)$ comes from a spherically symmetric solution. ${ }^{18}$ For the Kerr-Newman geometry $f(r)=m r-e^{2} / 2$, it can change the sign which leads to the causality violation related to regions where $g_{\phi \phi}<0$. Regular spherical solutions satisfy the weak energy condition, ${ }^{5}$ and $\mathcal{M}(r)$ is monotonically growing from 
$4 \pi \tilde{\rho}(0) r^{3} / 3$ as $r \rightarrow 0$ to $m-e^{2} / 2 r$ as $r \rightarrow \infty .^{22}$ As a result a function $f(r)=r \mathcal{M}(r)$ is non-negative and $g_{\phi \phi}>0$ in (9) which guarantees the causal safety on the whole manifold. The mass parameter $m=4 \pi \int_{0}^{\infty} \tilde{\rho}(r) r^{2} d r$ appearing in a spinning solution, is the finite positive electromagnetic mass, ${ }^{22}$ generically related to interior de Sitter vacuum for any solution from the class specified by (3). ${ }^{13}$

The surfaces of constant $r$ are the oblate confocal ellipsoids given by ${ }^{24}$

$$
r^{4}-\left(x^{2}+y^{2}+z^{2}-a^{2}\right) r^{2}-a^{2} z^{2}=0 .
$$

For $r=0$ they degenerate to the equatorial disk

$$
x^{2}+y^{2} \leq a^{2}, \quad z=0
$$

centered on the symmetry axis and bounded by the ring

$$
x^{2}+y^{2}=a^{2}, \quad z=0 .
$$

In the Kerr-Newman metric the ring (13) comprises the Kerr singularity of the Kerr-Newman geometry. ${ }^{24}$

The Cartesian coordinates $x, y, z$ are related to the Boyer-Lindquist coordinates $r, \theta, \phi$ by

$$
x^{2}+y^{2}=\left(r^{2}+a^{2}\right) \sin ^{2} \theta ; \quad z=r \cos \theta .
$$

The eigenvalues of the stress-energy tensor are calculated in the co-rotating frame where each of ellipsoidal layers rotates with the angular velocity $\omega(r)=u^{\phi} / u^{t}=$ $a /\left(r^{2}+a^{2}\right) \cdot{ }^{16}$ In the $\mathcal{R}$-regions inside the internal Cauchy horizon and outside the event horizon they are related to the function $f(r)$ by ${ }^{18}$

$$
\kappa \Sigma^{2} \rho=2\left(f^{\prime} r-f\right) ; \kappa \Sigma^{2} p_{\perp}=2\left(f^{\prime} r-f\right)-f^{\prime \prime} \Sigma .
$$

It follows

$$
\kappa \rho=\frac{r^{4}}{\Sigma^{2}} \tilde{\rho}(r) ; \quad \kappa p_{\perp}=\left(\frac{r^{4}}{\Sigma^{2}}-\frac{2 r^{2}}{\Sigma}\right) \tilde{\rho}(r)-\frac{r^{3}}{2 \Sigma} \tilde{\rho}^{\prime}(r) .
$$

The prime denotes the derivative with respect to $r$.

\section{Geometry}

\subsection{Horizons, ergospheres and ergoregions}

Space-time can have at most two horizons ${ }^{5}$ defined by $\Delta(r)=r^{2}+a^{2}-2 f(r)=0$. Ergoregions, where $g_{t t}<0$, exist between the event horizon and ergosphere (the static limit $\left.g_{t t}=0\right)$ given by $r^{2}+a^{2} \cos ^{2} \theta-2 f(r)=0$. Four possibilities for the existence of ergospheres and ergoregions are shown in Fig. 1.

The width of the ergosphere at a certain $z$ is $x^{2}+y^{2}=\left(a^{2}+r^{2}\right)\left(1-z^{2} / r^{2}\right)$. In the equatorial plane $x^{2}+y^{2}=a^{2}+r^{2}$ and $z^{2}=0=2 f(r)-r^{2}$. For any regular density profile the function $f(r)$ is everywhere non-negative. ${ }^{22}$ Ergosphere exists when the curve $u(r)=2 f(r)$ intersects or touches the parabola $u(r)=r^{2}$ (curve 2 

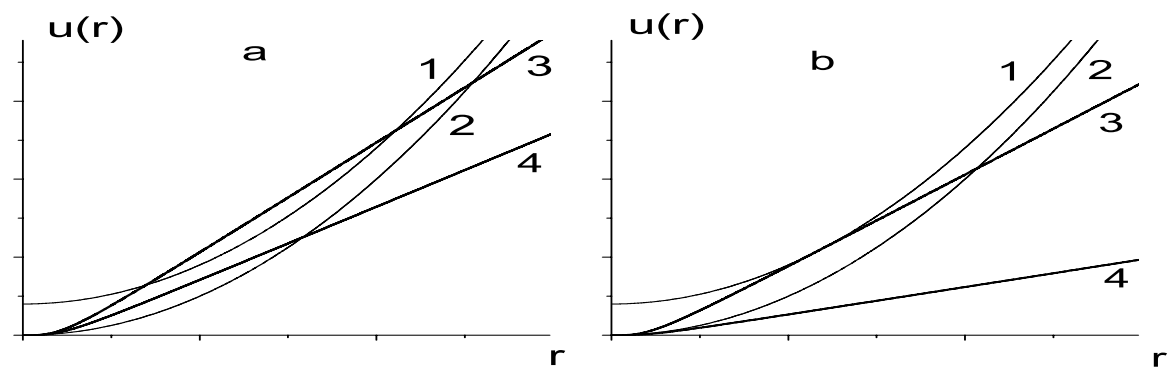

Fig. 1. Functions $2 f(r)$ and related parabolas (thin lines) for 4 cases of existence of ergospheres.

in Fig. 1). It is evident that in this case the curve $u(r)=2 f(r)$ intersects also the (situated above) parabola $u(r)=r^{2}+a^{2} \cos ^{2} \theta$ for a given $\theta$ (curve 1 in Fig. 1 ).

In the black hole case, ergospheres and ergoregions exist for any density profile (the curve $3 \mathrm{a}$ for the case of two horizons, the curve $3 \mathrm{~b}$ for the double-horizon case).

In the case of a soliton (a structure without horizons), existence of ergospheres depends on the density profile. Solitons can have two ergospheres and ergoregion between them (curve 4a), one ergosphere and ergoregion involving the whole interior (curve 4b), or no ergospheres. ${ }^{5}$

\subsection{De Sitter vacuum surfaces}

In the co-rotating frame we obtain from the equation (15)

$$
\kappa\left(p_{\perp}+\rho\right)=2\left(\frac{r^{4}}{\Sigma^{2}}-\frac{r^{2}}{\Sigma}\right) \tilde{\rho}(r)-\frac{r^{3}}{2 \Sigma} \tilde{\rho}^{\prime}(r) .
$$

In the equatorial plane $r^{2} / \Sigma \rightarrow 1$ as $z \rightarrow 0,{ }^{10}$ and the equation (17) reduces to ${ }^{5}$

$$
\kappa\left(p_{\perp}+\rho\right)=-r \tilde{\rho}^{\prime}(r) / 2
$$

For the spherically symmetric solutions regularity requires $r \tilde{\rho}^{\prime}(r) \rightarrow 0$ as $r \rightarrow 0 .^{22}$ As a result the equation (18) gives on the disk the equation of state

$$
p_{\perp}=p_{r}=-\rho,
$$

which represents the rotating de Sitter vacuum. By (16), the density in the equatorial plane is $\rho(r, \theta)=\tilde{\rho}(r)$. On the disk $\rho(r, \theta)=\tilde{\rho}(0)$.

The equation (17) can be written as

$$
\kappa\left(p_{\perp}+\rho\right)=\frac{2 r^{2}}{\Sigma^{2}}\left(\frac{\Sigma r}{4}\left|\tilde{\rho}^{\prime}\right|-\tilde{\rho} a^{2} \cos ^{2} \theta\right) .
$$


It implies a possibility of generic violation of the weak energy condition beyond the surface $\mathcal{E}(r, z)=0$ on which $p_{\perp}=-\rho$. It can be expressed through the pressure of a related spherical solution, $\tilde{p_{\perp}}=-\tilde{\rho}-r \tilde{\rho}^{\prime} / 2,{ }^{13}$ which gives $^{5}$

$$
\kappa\left(p_{\perp}+\rho\right)=\frac{r\left|\tilde{\rho}^{\prime}\right|}{2 \Sigma^{2}} \mathcal{E}(r, z) ; \mathcal{E}(r, z)=r^{4}-z^{2} P(r) ; P(r)=\frac{2 a^{2}}{r\left|\tilde{\rho}^{\prime}\right|}\left(\tilde{\rho}-\tilde{p_{\perp}}\right)
$$

The existence of de Sitter $\mathcal{E}$-surfaces is possible only for the mass functions originated from spherical solutions satisfying the dominant energy condition $\left(\tilde{\rho} \geq \tilde{p_{k}}\right)$.

Each point of the $\mathcal{E}$-surface belongs to some of confocal ellipsoids (11). In the Cartesian coordinates (14) the equation of the ellipsoid (11) reads $\left(r^{2}-z^{2}\right)\left(a^{2}+r^{2}\right)=$ $r^{2}\left(x^{2}+y^{2}\right)$. On the $\mathcal{E}$-surface we have $z^{2}=r^{4} / P(r)$. The squared width of the $\mathcal{E}$-surface, $W_{\mathcal{E}}^{2}=\left(x^{2}+y^{2}\right)_{\mathcal{E}}=\left(a^{2}+|z| \sqrt{P(r)}\right)(1-|z| / \sqrt{P(r)})$. The $\mathcal{E}$-surface is entirely confined within the $r_{*}$-ellipsoid whose minor axis coincides with $|z|_{\text {max }}$ for the $\mathcal{E}$-surface. ${ }^{5}$ For regular solutions $r \tilde{\rho}^{\prime} \rightarrow 0, \quad p_{\perp} \rightarrow-\rho$ as $r \rightarrow 0,{ }^{22}$ and $P(r) \rightarrow A^{2} r^{-(n+1)}$ as $r \rightarrow 0$, with the integer $n \geq 0$. As a result the derivative of $W_{\mathcal{E}}(z)$ near $z \rightarrow 0$ behaves as $z^{-(n+1) /(n+5)}$ and goes to $\pm \infty$ as $z \rightarrow 0$, so that the function $W_{\mathcal{E}}(z)$ has the cusp at approaching the disk and two symmetric (with respect to the equatorial plane) maxima between $z= \pm r_{*}$ and $z=0 .{ }^{5}$

The $\mathcal{E}$-surface is plotted below for the regularized Coulomb profile ${ }^{22} \tilde{\rho}=$ $q^{2} /\left(r^{2}+r_{q}^{2}\right)^{2} ; \quad r_{q}=\pi q^{2} / 8 m$. For this profile $P\left(r_{*}\right)=r_{*}^{2}=r_{q} a$, and the $\mathcal{E}$-surface is given by $\mathcal{E}(r, z)=r^{6}-r_{*}^{4} z^{2}=0$. Relation between the width of the $\mathcal{E}$-surface in the equatorial plane $W_{\mathcal{E}}=a$ and its height $|z|_{\text {max }}=\sqrt{a r_{q}}$ defines the explicit form of the $\mathcal{E}$-surface. It depends on two parameters: $\alpha=a / m$ and specific charge $\beta=q / \mathrm{m}$. For black holes the parameter $\beta$ changes within the range $0<\beta<0.99$. $^{25,26}$ The height of the $\mathcal{E}$-surface $H_{\mathcal{E}}=\sqrt{\frac{a}{r_{q}}}=\frac{2 \sqrt{2 \alpha}}{\sqrt{\pi} \beta}$ and its width $W_{\mathcal{E}}=\frac{a}{r_{q}}=\frac{8 \alpha}{\pi \beta^{2}}$. For $\alpha<\pi \beta^{2} / 8$ we have $H_{\mathcal{E}} / W_{\mathcal{E}}>1$ and the $\mathcal{E}$-surface is prolate (Fig. 2). It can be the case of a slowly rotating moderately charged black hole.

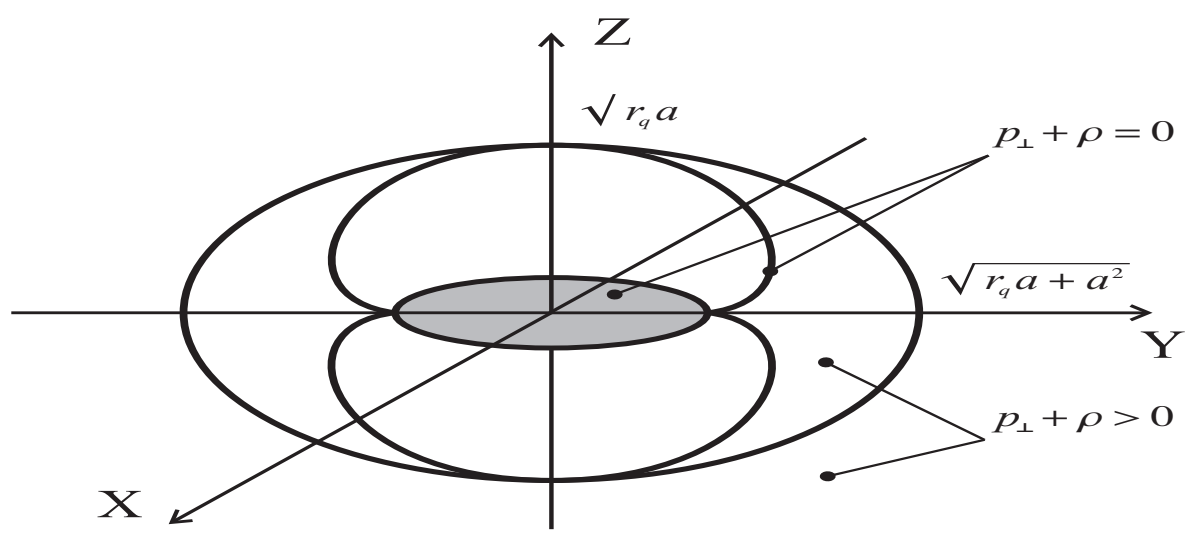

Fig. 2. Vacuum $\mathcal{E}$-surface for the case $\alpha<\pi \beta^{2} / 8$. 


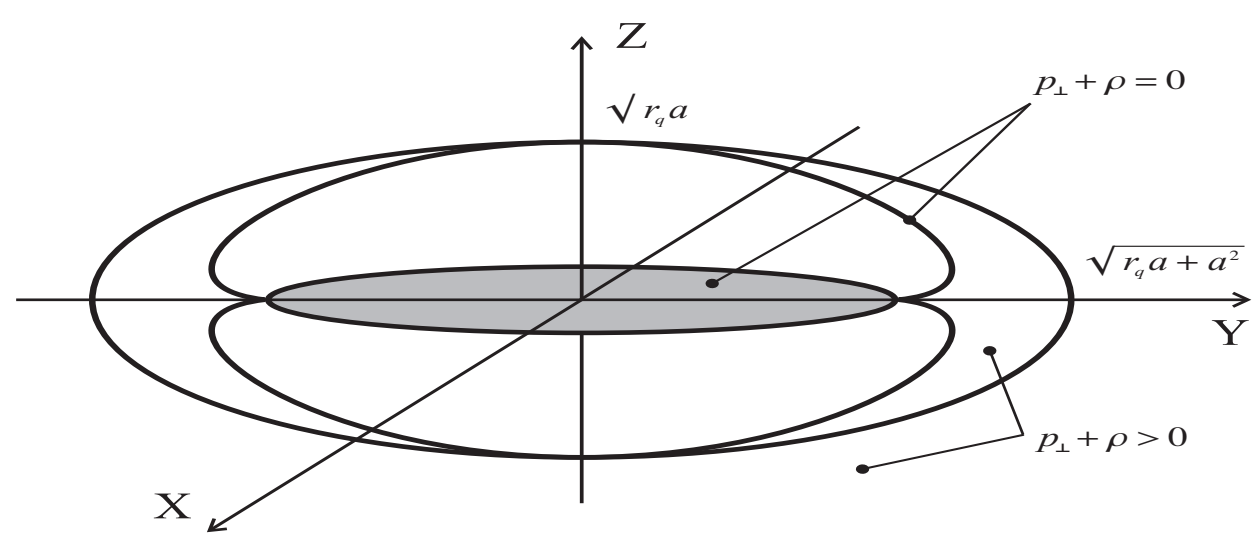

Fig. 3. Vacuum $\mathcal{E}$-surface for the case $\alpha>\pi \beta^{2} / 8$.

In the case $\alpha>\pi \beta^{2} / 8$, the $\mathcal{E}$-surface is oblate (Fig. 3 ). It is the case for electromagnetic spinning soliton. It can be also the case of a slightly charged rotating black hole and of an extreme black hole.

\section{Generic Interiors}

\subsection{Electromagnetic fields}

In the axially symmetric case non-zero field components are $F_{01}, F_{02}, F_{13}, F_{23}$. In geometry $(9)$ they are related by $F_{31}=a \sin ^{2} \theta F_{10} ; a F_{23}=\left(r^{2}+a^{2}\right) F_{02}$.

The relation connecting fields with density and pressure reads ${ }^{10}$

$$
\kappa\left(p_{\perp}+\rho\right)=2 \mathcal{L}_{F}\left(F_{10}^{2}+\frac{F_{20}^{2}}{a^{2} \sin ^{2} \theta}\right) .
$$

The dynamical equations $\nabla_{\mu}\left(\mathcal{L}_{F} F^{\mu \nu}\right)=0$ are satisfied by the functions ${ }^{10}$

$$
F_{10}=\frac{q}{\Sigma^{2} \mathcal{L}_{F}}\left(r^{2}-a^{2} \cos ^{2} \theta\right) ; F_{02}=\frac{q}{\Sigma^{2} \mathcal{L}_{F}} a^{2} r \sin 2 \theta
$$

It results in

$$
\kappa\left(p_{\perp}+\rho\right)=\frac{2 q^{2}}{\mathcal{L}_{F} \Sigma^{2}}
$$

Equation of state $p=-\rho$ on the disk and the $\mathcal{E}$-surface dictated by geometry for regular solutions, requires $\mathcal{L}_{F} \Sigma^{2} \rightarrow \infty$ and hence $\mathcal{L}_{F} \rightarrow \infty$. In this limit dynamical equations $\nabla_{\mu}\left(\mathcal{L}_{F} F^{\mu \nu}\right)=0$ are compatible with equations $\nabla_{\mu}{ }^{*} F^{\mu \nu}=0 .^{5}$

The field vectors

$$
\mathbf{E}=\left\{F_{0 \alpha}\right\} ; \mathbf{D}=\left\{\mathcal{L}_{F} F^{0 \alpha}\right\} ; \mathbf{B}=\left\{{ }^{*} F^{\alpha 0}\right\} ; \mathbf{H}=\left\{\mathcal{L}_{F}{ }^{*} F_{0 \alpha}\right\}
$$


satisfy the field equations in the Maxwell form $\nabla \mathbf{D}=0 ; \quad \nabla \times \mathbf{H}=\frac{\partial \mathbf{D}}{\partial t} ; \quad \nabla \mathbf{B}=0$; $\nabla \times \mathbf{E}=-\frac{\partial \mathbf{B}}{\partial t}$. Field intensities are connected with the field inductions as

$$
D_{\alpha}=\epsilon_{\alpha \beta} E_{\beta} ; \quad B_{\alpha}=\mu_{\alpha \beta} H_{\beta}
$$

and the tensors of the electric and magnetic permeability read ${ }^{10}$

$$
\epsilon_{r}=\frac{\left(r^{2}+a^{2}\right)}{\Delta} \mathcal{L}_{F} ; \quad \epsilon_{\theta}=\mathcal{L}_{F} ; \quad \mu_{r}=\frac{\left(r^{2}+a^{2}\right)}{\Delta} \frac{1}{\mathcal{L}_{F}} ; \quad \mu_{\theta}=\frac{1}{\mathcal{L}_{F}} .
$$

In the de Sitter regions $\epsilon_{r}=\epsilon_{\theta}=\mathcal{L}_{F} \rightarrow \infty ; \mu_{r}=\mu_{\theta}=\mathcal{L}_{F}^{-1}=0$, so that the de Sitter vacuum surfaces display properties of a perfect conductor and ideal diamagnetic.

De Sitter vacuum $\mathcal{E}$-surface contains de Sitter disk as the bridge, $p_{\perp}+\rho=0$ on the whole surface, but in cavities between its upper and down boundaries the weak energy condition (WEC) can be violated.

For spherical NED-GR solutions WEC is always satisfied since $\kappa\left(p_{\perp}+\rho\right)=$ $-\mathcal{L}_{F} F$, where $F \leq 0$ and $\mathcal{L}_{F}$ is the electric permeability ${ }^{22}$ which should be positive in electrodynamics of continued media.

Violation of WEC was reported for regular solutions obtained with the NewmanJanis algorithm from the Hayward and Bardeen metrics ${ }^{27}$ and from the Ayón-BeatoGarcia solution, ${ }^{28}$ and for regular solutions obtained by postulating a metric $g_{\mu \nu}$ and calculating $T_{\mu \nu}$ from the Einstein equations. ${ }^{29}$

In the NED-GR electrically charged objects a negative value of $\left(p_{\perp}+\rho\right)$ in (22) would mean negative values for the electric and magnetic permeabilities inadmissible in electrodynamics of continued media. ${ }^{30}$

The alternative compatible with regularity is zero value of $p_{\perp}+\rho$ also inside $\mathcal{E}$ surface. This can be the case for the shell-like models with the flat vacuum interior, zero fields and hence zero density and pressures, ${ }^{31}$ and for bag-like models. ${ }^{32}$

Another possibility favored by the underlying idea of non-linearity regularizing a singularity and suggested by vanishing magnetic induction on the vacuum $\mathcal{E}$ surface, ${ }^{5}$ is extension of its basic property $\left(p_{\perp}=-\rho\right)$ to its interior. Then the regular interior is presented by the de Sitter vacuum core, and the weak energy condition is valid in the whole core.

\subsection{Elementary superconductivity}

The magnetic induction $\mathbf{B}$ vanishes on the disk,, 10 as well as the magnetic permeability $\mu$. In electrodynamics of continued media the transition to a superconducting state corresponds to the limits $\mathbf{B} \rightarrow 0$ and $\mu \rightarrow 0$ in a surface current $\mathbf{j}_{\mathbf{s}}=\frac{(1-\mu)}{4 \pi \mu}[\mathbf{n B}]$, where $\mathbf{n}$ is the normal to the surface. The right-hand side then becomes indeterminate, and there is no condition which would restrict the possible values of the current. ${ }^{30}$ In the strongly nonlinear electromagnetic field on the disk $\mu=\mathcal{L}_{F}^{-1}$ but B also depends on $\mathcal{L}_{F}$, so we have to calculate precise formula defining the surface current on the disk. We apply definition of a surface current for a charged surface layer, $4 \pi j_{k}=\left[e_{(k)}^{\alpha} F_{\alpha \beta} n^{\beta}\right],{ }^{6}$ where $[.$.$] denotes a jump across the layer; e_{(k)}^{\alpha}$ are the 
base vectors associated with the intrinsic coordinates on the disk $t, \phi, 0 \leq \xi \leq \pi / 2$; $n_{\alpha}=\left(1+q^{2} / a^{2}\right)^{-1 / 2} \cos \xi \delta_{\alpha}^{1}$ is the unit normal directed upwards. ${ }^{6}$ With using (23) and $(27)$ we obtain the surface current ${ }^{20}$

$$
j_{\phi}=-\frac{q}{2 \pi a} \sqrt{1+q^{2} / a^{2}} \sin ^{2} \xi \frac{\mu}{\cos ^{3} \xi} .
$$

At approaching the $\operatorname{ring} r=0, \xi=\pi / 2$, both terms in the second fraction go to zero quite independently. As a result the surface currents on the ring can be any and amount to a non-zero total value. Superconducting current flowing (forever) on the de Sitter vacuum ring serves as a source of the Kerr-Newman fields. This kind of a source is non-dissipative so that life time of an object can be unlimited.

\section{Summary and Discussion}

We outline the basic generic properties of regular rotating electrically charged rotating black holes and solitons in nonlinear electrodynamics minimally coupled to gravity which follow from the analysis of the source-free NED-GR dynamical equations, in which nonlinear electromagnetic field provides a source in the Einstein equations. They are described by the NED-GR solutions asymptotically Kerr-Newman for a distant observer. Black holes have at most two horizons and ergoregions. Solitons can have two ergospheres and ergoregion between them, one ergosphere and ergoregion involving the whole interior, or no ergospheres.

Interiors include de Sitter vacuum surfaces $p_{\perp}+\rho=0$ with de Sitter disk $r=0$ as the bridge. Disk is confined by the regular ring replacing the Kerr singularity. A superconducting current flowing on the ring serves as a non-dissipative source of the Kerr-Newman exterior fields, providing unlimited life time of the NED-GR regular spinning objects. ${ }^{20}$

In the spirit of the Dirac extended electron, ${ }^{33}$ with the Carter remarkable discovery of ability of the Kerr-Newman solution to represent the electron for a distant observer, electromagnetic spinning soliton can be considered as the Coleman lump (a non-singular, non-dissipative solution of finite energy holding itself together by its own self-interaction $\left.{ }^{34}\right)$. For the electron $q=-e, a=\lambda / 2$ and $\lambda=\hbar / m$ is its Compton wavelength. ${ }^{3}$ In the observer region $r \gg \lambda$

$$
\begin{gathered}
E_{r}=-\frac{e}{r^{2}}\left(1-\frac{\hbar^{2}}{m_{e}^{2} c^{2}} \frac{3 \cos ^{2} \theta}{4 r^{2}}\right) ; \quad E_{\theta}=\frac{e \hbar^{2}}{m_{e}^{2} c^{2}} \frac{\sin 2 \theta}{4 r^{3}} \\
B^{r}=-\frac{e \hbar}{m_{e} c} \frac{\cos \theta}{r^{3}} ; \quad B_{\theta}=-\frac{e \hbar}{2 m_{e} c} \frac{\sin \theta}{r^{4}}
\end{gathered}
$$

The Planck constant appears due to discovered by Carter ability of the KerrNewman solution to present the electron as seen by a distant observer. In terms of the Coleman lump equation (30) describes the following situation: The leading term in $E_{r}$ gives the Coulomb law as the classical limit $\hbar=0$, and the higher terms represent the quantum corrections. 
Purely electromagnetic reaction of annihilation $e^{+} e^{-} \rightarrow \gamma \gamma(\gamma)$ reveals, at the $5 \sigma$ confidence level, the existence of the minimal length $l_{e}=1.57 \times 10^{-17} \mathrm{~cm}$ at the scale $E=1.253 \mathrm{TeV}$ which can be explained as the distance of the closest approach of annihilating particles where electromagnetic attraction is stopped by the gravitational repulsion of the interior de Sitter vacuum. ${ }^{35}$

Application of the Casimir invariants of the de Sitter group in the region surrounding the interaction vertex for the sub-eV particles, predicts $\mathrm{TeV}$ scale for gravitoelectroweak unification and explains the experimental results known as a negative mass-squared difference for neutrino. ${ }^{36}$

\section{References}

1. E. T. Newman, E. Cough, K. Chinnapared, A. Exton, A. Prakash and R. Torrence, J. Math. Phys. 6, 918 (1965).

2. E. T. Newman and A. J. Janis, J. Math. Phys. 6, 915 (1965).

3. B. Carter, Phys. Rev. 174, 1559 (1968).

4. A. Burinskii, Int. J. Mod. Phys. A 29, 1450133 (2014).

5. I. Dymnikova and Galaktionov, Class. Quant. Grav. 32, 165015 (2015).

6. W. Israel, Phys. Rev. D 2, 641 (1970).

7. E. S. Fradkin and A. A. Tseytlin, Phys. Lett. B 163, 123 (1985).

8. A. A. Tseytlin, Nucl. Phys. B 276, 391 (1986).

9. N. Siberg and E. Witten, JHEP 1999, 032 (1999).

10. I. Dymnikova, Phys. Lett. B 639, 368 (2006).

11. I. Dymnikova, Gen. Rel. Grav. 24, 235 (1992).

12. I. Dymnikova, Phys. Lett. B 472, 33 (2000).

13. I. Dymnikova, Class. Quant. Grav. 19, 725 (2002).

14. I. Dymnikova, Int. J. Mod. Phys. D 12, 1015 (2003).

15. R. P. Kerr and A. Schild, Proc. of Symp. in Appl. Math. 17, 199 (1965).

16. A. Burinskii, E. Elizalde, S. R. Hildebrandt and G. Magli, Phys. Rev. D 65, 064039 (2002).

17. E. Elizalde and S. R. Hildebrandt, Phys. Rev. D 65, 124024 (2002).

18. M. Gürses and F. Gürsey, J. Math. Phys. 16, 2385 (1975).

19. A. Burinskii and S. R. Hildebrandt, Phys. Rev. D 65, 104017 (2002).

20. I. Dymnikova, Journal of Gravity 2015, 904171 (2015).

21. K. A. Bronnikov, Phys. Rev. D 63, 044005 (2001).

22. I. Dymnikova, Class. Quant. Grav. 21, 4417 (2004).

23. I. Dymnikova, E. Galaktionov and E. Tropp, Adv. in Math. Phys. 2015, 496475 (2015).

24. S. Chandrasekhar, The Mathematical Theory of Black Holes (Clarendon, Oxford, 1992).

25. M. Zilhão, V. Cardoso, C. Herdeira, L. Lehner and U. Sperhake, Phys. Rev. D 89, 044008 (2014).

26. A. J. S. Hamilton, Phys. Rev. D 84, 124057 (2011).

27. C. Bambi and L. Modesto, Phys. Lett. B 721, 329 (2013).

28. B. Toshmatov, B. Ahmedov, A. Abdujabbarov and Z. Stuchlik, Phys. Rev. D 89, 104017 (2014).

29. J. C. S. Neves and A. Saa, Phys. Lett. D 734, 44 (2014).

30. L. D. Landau and E. M. Lifshitz, Electrodynamics of Continued Media (Pergamon Press, Oxford, 1993).

31. C. A. Lopez, Phys. Rev. D 30, 313 (1984). 
32. A. Burinskii, Zh. Exp. Teor. Fiz. 148, 228 (2015).

33. P. A. M. Dirac, Proc. Roy. Soc. Lond. A 268, 57 (1962).

34. S. Coleman, in New Phenomena in Subnuclear Physics, ed. A. Zichichi (Plenum, New York, 1977), p. 297.

35. I. Dymnikova, A. Sakharov and J. Ulbricht, AHEP 2014, 707812 (2014).

36. D. V. Ahluwalia and I. Dymnikova, Intern. J. Mod. Phys. D 12, 1878 (2003). 